\title{
The lattice infrared Landau gauge gluon propagator: from finite volume to the infinite volume
}

\section{O. Oliveira*1, P. J. Silva ${ }^{1,2}$}

${ }^{1}$ Dep. Física, Universidade de Coimbra, 3004-516 Coimbra, Portugal

2 School of Physics and Astronomy, University of Edinburgh, Edinburgh EH9 3JZ, UK

E-mail: orlando@teor.fis.uc.pt, psilva@teor.fis.uc.pt

The Landau gauge lattice gluon propagator is discussed for different sets of lattices. Particular attention is given to its infrared properties. Our results show that the lattice propagator can be made compatible with either the decoupling-like or the scaling-like solution of the Dyson-Schwinger equations. Furthermore, the analysis of the Cucchieri-Mendes bounds is performed considering large volume simulations and the Oliveira-Silva ratios are computed. If the first do not give a clear answer about the value of $D(0)$, the second method favors a $D(0)=0$. Finally, the SU(3) and $\mathrm{SU}(2)$ propagators are compared in the infrared. It comes out that the propagators are different although the infrared exponents seem to be similar. The analysis suggests a scaling behaviour $D(0) \sim N$ with the gauge group $\mathrm{SU}(\mathrm{N})$.

International Workshop on QCD Green's Functions, Confinement, and Phenomenology - QCD-TNT09 September 07 - 112009

ECT Trento, Italy

\footnotetext{
${ }^{*}$ Speaker.
} 


\section{Introduction}

For a given quantum field theory the Green's functions encode the dynamical information. For QCD, in particular, the computation of any Green's function, such as the gluon propagator $D\left(q^{2}\right)$, over the entire momentum spectrum cannot rely on perturbation theory. In what concerns the gluon propagator, in the past years, there has been a discussion about its infrared behaviour and its value at zero momentum in the Landau gauge. The recent effort on computing $D(0)$ comes from its relation with the Gribov-Zwanziger gluon confinement mechanism, which implies $D(0)=0$, and with the possibility of dynamical mass generation for gluons. The dispute is still going on and involves both Schwinger-Dyson solutions and lattice QCD results.

The recent solutions of the Dyson-Schwinger equations have different infrared behaviours. On one side we have the scaling solution $[1,2]$ with $D(0)=0$ and an infrared behaviour given by a pure power law $D\left(q^{2}\right)=\left(q^{2}\right)^{2 \kappa-1}$, with $\kappa \sim 0.595$. On the other side we have the decoupling solution $[3,4]$ (see also [5]) with a finite and nonvanishing $D(0)$, with the value of $D(0)$ being related with a dynamical generated gluon mass, and a plateau for $D\left(q^{2}\right)$ at low momenta.

The recent lattice simulations also show contradictory results. Indeed, large volume simulations using the Wilson action and large lattice spacings, i.e. $a \sim 0.18 \mathrm{fm}$ or larger, show a gluon propagator that agrees qualitatively with the decoupling solution. However, as we will see, a second look at the lattice data seems to indicate that the solution $D(0)=0$ is still compatible with the lattice data - see below.

Here, we want to discuss on what the lattice simulations tell us about the infrared gluon propagator and, hopefully, point towards a "favorite" value of $D(0)$. In the following, we will use standard definitions for each of the quantities, which will not be shown here. The interested reader can find the details for example in [6].

\section{Lattice setup}

Our discussion of the infrared gluon propagator in the Landau gauge uses lattice data for the $S U$ (3) gluon propagator from simulations with two different values of $\beta$, namely $\beta=6.0$ and $\beta=5.7$, combining data generated at Coimbra with the data from the Berlin-Moscow-Adelaide group [8]. Furthermore, the SU(3) data will be compared with the large volumes, i.e. $80^{4}$ and $128^{4}$ at $\beta=2.2, \mathrm{SU}(2)$ propagator of the $\mathrm{S}$. Carlos group [9].

The lattices simulated using the SU(3) gauge group and the number of configurations for $\beta=5.7$ are

\begin{tabular}{ccccccccccccc}
\hline $\mathrm{L}$ & 8 & 10 & 14 & 18 & 26 & 36 & 44 & $64^{*}$ & $72^{*}$ & $80^{*}$ & $88^{*}$ & $96^{*}$ \\
$\mathrm{~L}(\mathrm{fm})$ & 1.5 & 1.8 & 2.6 & 3.3 & 4.8 & 6.6 & 8.1 & 11.8 & 13.2 & 14.7 & 16.2 & 17.6 \\
\# Confs & 56 & 149 & 149 & 149 & 132 & 100 & 29 & 14 & 20 & 25 & 68 & 67 \\
\hline
\end{tabular}

where * stands for simulations carried out by the Berlin-Moscow-Adelaide group [8]. Note that we took the lattice spacing from the string tension [7] and not from $r_{0}$ as the Berlin-Moscow-Adelaide group did in [8]. We have rescaled their data according to our definitions. We would like to call the reader's attention that different volumes have different statistics. The lattice gluon propagator for $\beta=5.7$ can be seen in figure 1 . 


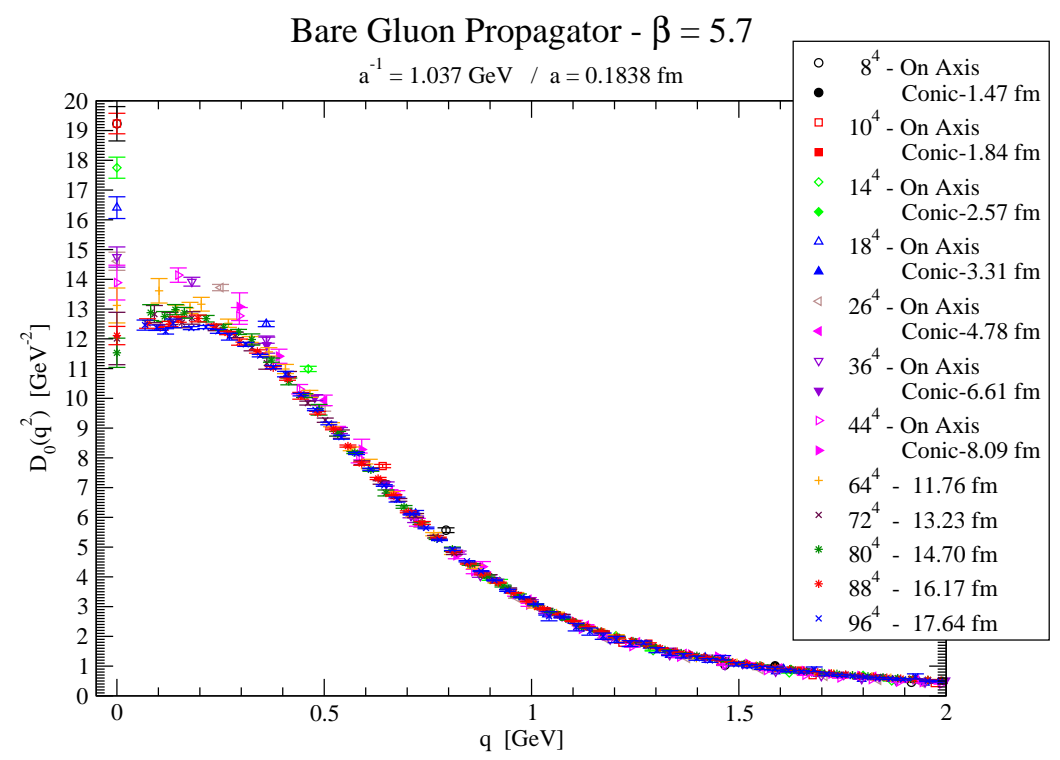

Figure 1: Bare gluon propagator from $\beta=5.7$ simulations. The data for the largest volumes at $L \geq 64$ is the Berlin-Moscow-Adelaide data [8] rescaled to our definition of the lattice spacing.

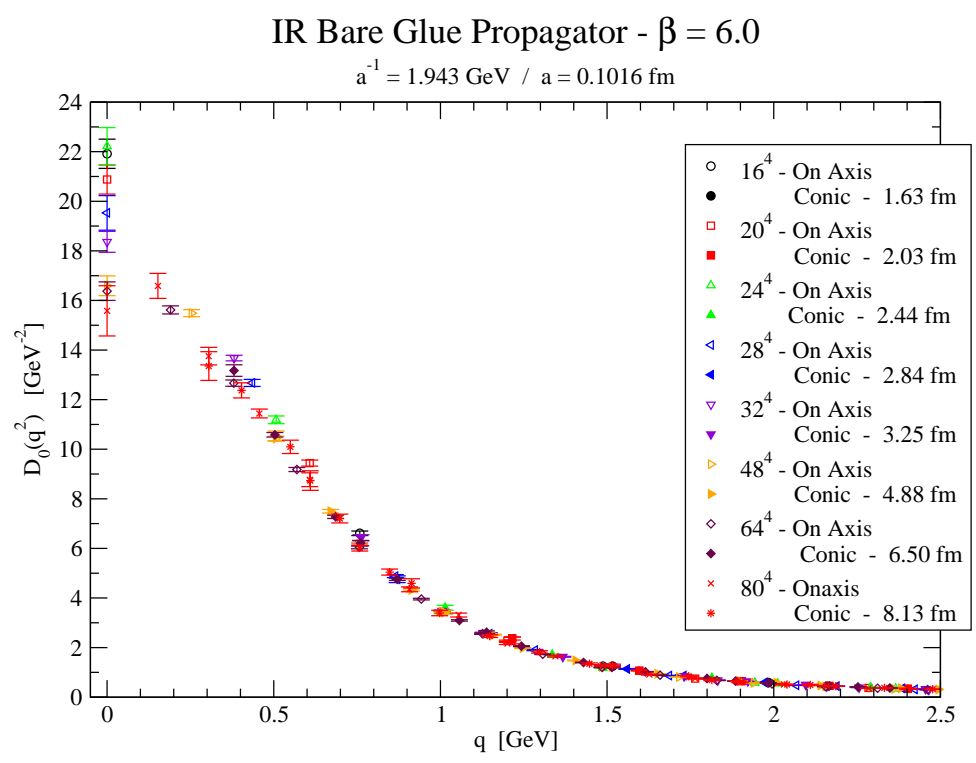

Figure 2: Bare gluon propagator from $\beta=6.0$ simulations.

The simulations for $\beta=6.0$, where the lattice spacing is $a=0.1016(25) \mathrm{fm}$, were carried for the following lattices

and the propagators are plotted in figure 2.

As seen in figures 1 and 2, no supression of the gluon propagator is observed. Therefore, as a first and naive conclusion one could claim that, modulo finite volume effects, the zero momentum gluon propagator is finite and non-zero. 


\begin{tabular}{ccccccccc}
\hline $\mathrm{L}$ & 16 & 20 & 24 & 28 & 32 & 48 & 64 & 80 \\
$\mathrm{~L}(\mathrm{fm})$ & 1.6 & 2.0 & 2.4 & 2.8 & 3.2 & 4.9 & 6.5 & 8.1 \\
\# Confs & 52 & 72 & 60 & 56 & 126 & 104 & 120 & 18 \\
\hline
\end{tabular}

\section{Modelling The Gluon Propagator}

As a first step towards trying to distinguish between a vanishing or nonvanishing $D(0)$, we look at the compatibility of the lattice data with the functional forms which have been used to describe the two Dyson-Schwinger solutions, i.e. the infrared lattice data is fitted to

$$
D\left(q^{2}\right)=\frac{Z}{q^{2}+M^{2}},
$$

where $M^{2}$ plays the role of a hard mass, and

$$
D\left(q^{2}\right)=Z \frac{\left(q^{2}\right)^{2 \kappa-1}}{\left(q^{2}+\Lambda^{2}\right)^{2 \kappa}} .
$$

In the fits to (3.2) the point $q=0$ is not included. This is not unwise since finite volume effects are certainly larger for $D(0)$.

In what concerns the fits to the $\beta=5.7$ data, we have observed that for the massive like propagator (3.1), the smaller lattices $(L<6 \mathrm{fm})$ are not described by the above functional form. Moreover, for the largest lattices, (3.1) reproduces well the lattice data, i.e. fits have $\chi^{2} /$ d.o.f. $<$ 1.8 , for momenta up to $500 \mathrm{MeV}$ with a gluon mass in the range of $719(18) \mathrm{MeV}$ to $2.20(63) \mathrm{GeV}$. $M$ depends on the fitting range and on the lattice volume. It was observed that $M$ (and $Z$ ) tend to increase with the lattice volume. For the fits to (3.2), the results are similar. Indeed, the lattice data is well described by (3.2) to momenta up to $500 \mathrm{MeV}$, with $\Lambda$ decreasing from $\sim 900 \mathrm{MeV}$ to $\sim 420 \mathrm{MeV}$ as the lattice volume increases. The $\kappa$ is, within one standard deviation, above 0.5 suggesting that $D(0)=0$. Typical fits can be seen in figures 3 and 4 .

We repeated the procedure using the $\mathrm{SU}(2)$ gluon propagator of [9] and found a similar behaviour. The main difference being that the $\mathrm{SU}(2)$ mass scales are typically larger than the corresponding SU(3) mass scales. For $M$ we get numbers around $1 \mathrm{GeV}$, while $\Lambda$ stays between 700 $\mathrm{MeV}$ to $800 \mathrm{MeV}$ and $\kappa$ touch values slightly below the "magics" 0.5 .

The results of fitting the $\beta=6.0$ lattice gluon data give similar results. For completeness we resume the results as follows:

1. "Decoupling" Fit:

$48^{4}(\mathrm{~L}=4.9 \mathrm{fm}), q$ up to $508 \mathrm{MeV} ; M$ goes from $600(20) \mathrm{MeV}$ down to $578(12) \mathrm{MeV}$ as the fitting range is increased;

$64^{4}(\mathrm{~L}=6.5 \mathrm{fm}), q$ up to $503 \mathrm{MeV} ; M$ goes from $753(57) \mathrm{MeV}$ down to 655.8(9.3) MeV as the fitting range is increased;

$80^{4}(\mathrm{~L}=8.1 \mathrm{fm}), q$ up to $664 \mathrm{MeV} ; M$ goes from 588(129) $\mathrm{MeV}$ down to 576.4(6.6) $\mathrm{MeV}$ as the fitting range is increased; 


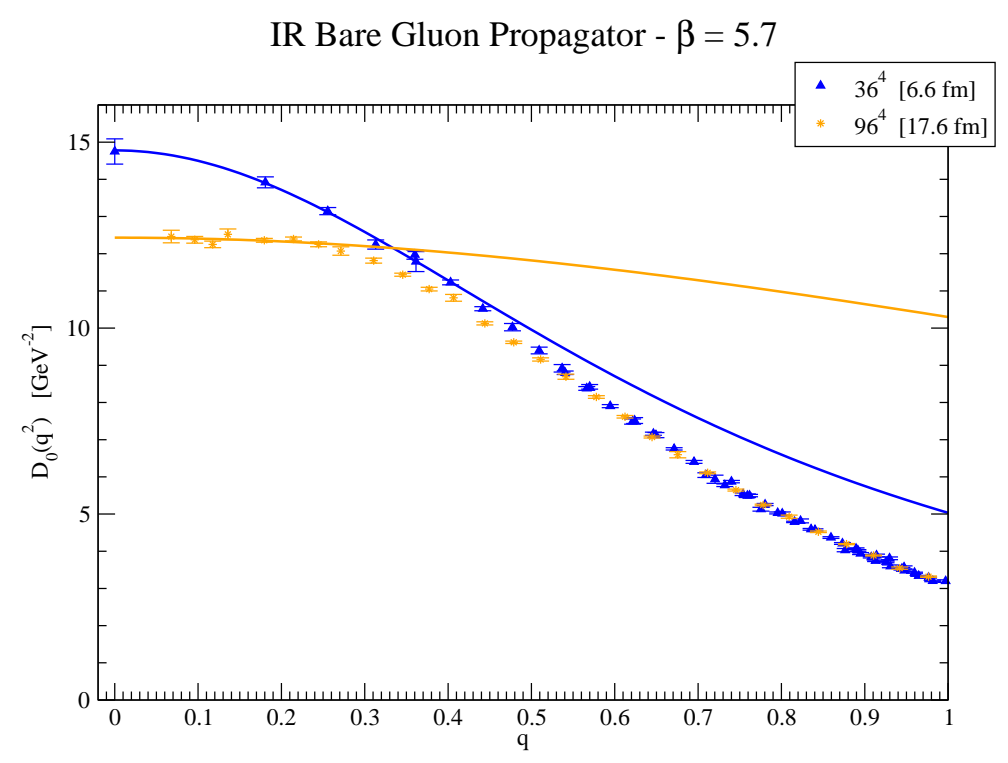

Figure 3: IR bare gluon propagator for $\beta=5.7$ and fits to (2.1).

\section{IR Bare Gluon Propagator $-\beta=5.7$}

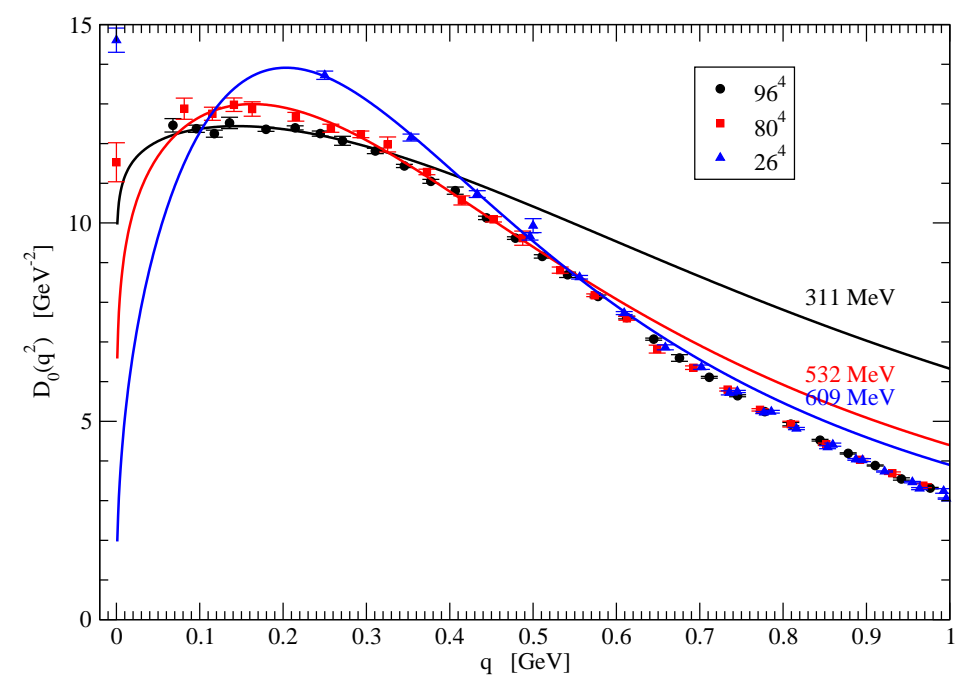

Figure 4: IR bare gluon propagator for $\beta=5.7$ and fits to (2.2). The MeV scales in the graph show the maximum fitting range compatible, i.e. with a $\chi^{2} /$ d.o.f. $<1.8$, with (2.2). Note that for the largest volume the $q=0 \mathrm{GeV}$ point is missing.

\section{2. "Scaling" Fit:}

$48^{4}(\mathrm{~L}=4.9 \mathrm{fm}), q$ up to $671 \mathrm{MeV} ; \Lambda$ goes from 460(61) MeV down to 432(27) $\mathrm{MeV}$ and $\kappa$ goes from $0.579(56)$ to $0.606(34)$ as the fitting range is increased;

$64^{4}(\mathrm{~L}=6.5 \mathrm{fm}), q$ up to $503 \mathrm{MeV} ; \Lambda$ goes from $609(111) \mathrm{MeV}$ up to 614(43) $\mathrm{MeV}$ and $\kappa$ goes from $0.510(31)$ to $0.513(16)$ as the fitting range is increased;

$80^{4}(\mathrm{~L}=8.1 \mathrm{fm}), q$ up to $645 \mathrm{MeV} ; \Lambda$ goes from $1.1(1.8) \mathrm{GeV}$ down to 582(54) $\mathrm{MeV}$ and $\kappa$ 


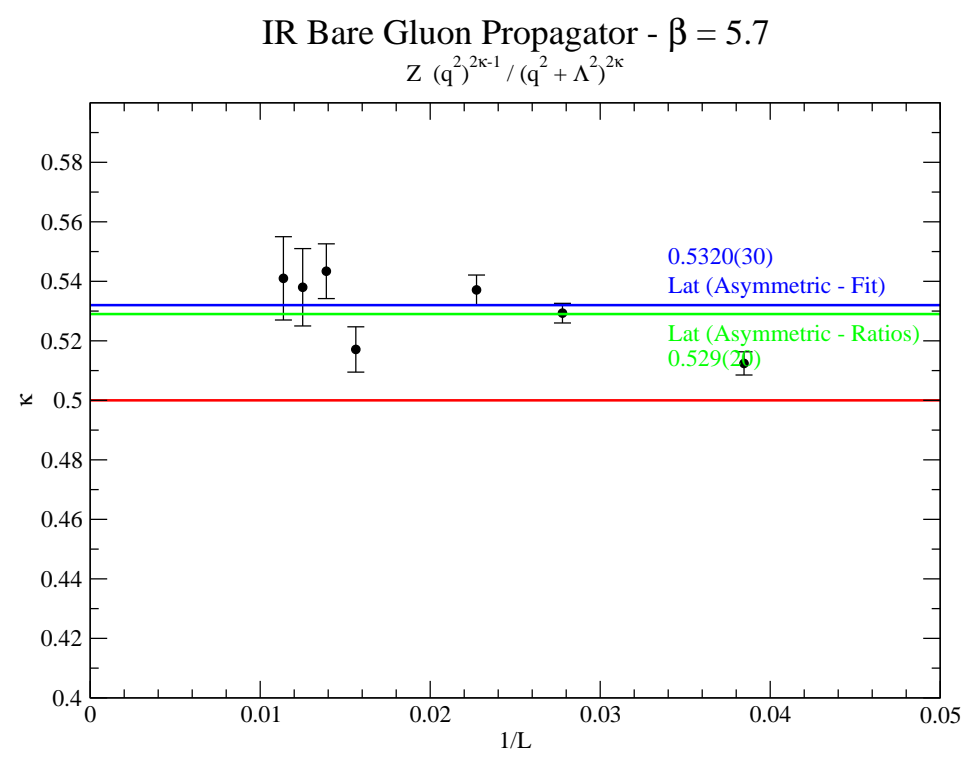

Figure 5: Exponent $\kappa$ as measured from fitting (2.2) (black points). The plot also shows $\kappa$ measured using $\beta=6.0$ asymmetric lattice data to fit (2.2) (blue line) and ratios of propagators (green line) using the same asymmetric lattice set. The red line is $\kappa=0.5$ and a value of $\kappa$ above 0.5 implies $D(0)=0$, a $\kappa<0.5$ means $D(0)=\infty$ and $\kappa=0.5$ implies a decoupling type solution.

goes from $0.453(61)$ to $0.556(17)$ as the fitting range is increased ${ }^{1}$.

From the previous analysis, the conclusion is that although a "decoupling" like gluon propagator seems to be favoured by the raw lattice data, a "scaling" like propagator is not excluded yet. The fits just described show that both type of solutions are in good agreement with the lattice data and that if a "scaling" like propagator is the solution, $D\left(q^{2}\right)$ starts to be supressed only for rather low momenta. Believing on the results of figure $4, D\left(q^{2}\right)$ is supressed only for momenta well below $100 \mathrm{MeV}$.

\section{Cucchieri-Mendes Bounds}

In [10], the authors derived inequalities between $D(0) / V$ and what they called an average absolute value of the components of the colour magnetization $M(0)$,

$$
\langle M(0)\rangle^{2} \leq \frac{D(0)}{V} \leq d\left(N_{c}^{2}-1\right)\left\langle M(0)^{2}\right\rangle
$$

where $d$ is the number of space-time dimensions and $N_{c}$ the number of colours. For the definition of $M(0)$ see the cited work. In the above expression, $\langle\cdots\rangle$ means Monte Carlo average and (4.1) follows directly from using a Monte Carlo approach.

In [10] the different terms in (4.1) were computed for SU(2) simulations and, after performing a scaling analysis, the authors conclude in favour of a finite and nonvanishing $D(0)$. Indeed, they

\footnotetext{
${ }^{1}$ The reader should be aware that the presented results for the $80^{4}$ lattice have a quite small statistics. So far, the total number of gauge configurations is 18 , which could explain the relative large errors in $\Lambda$ and the rather low value for $\kappa$ obtained with the smallest fitting range.
} 


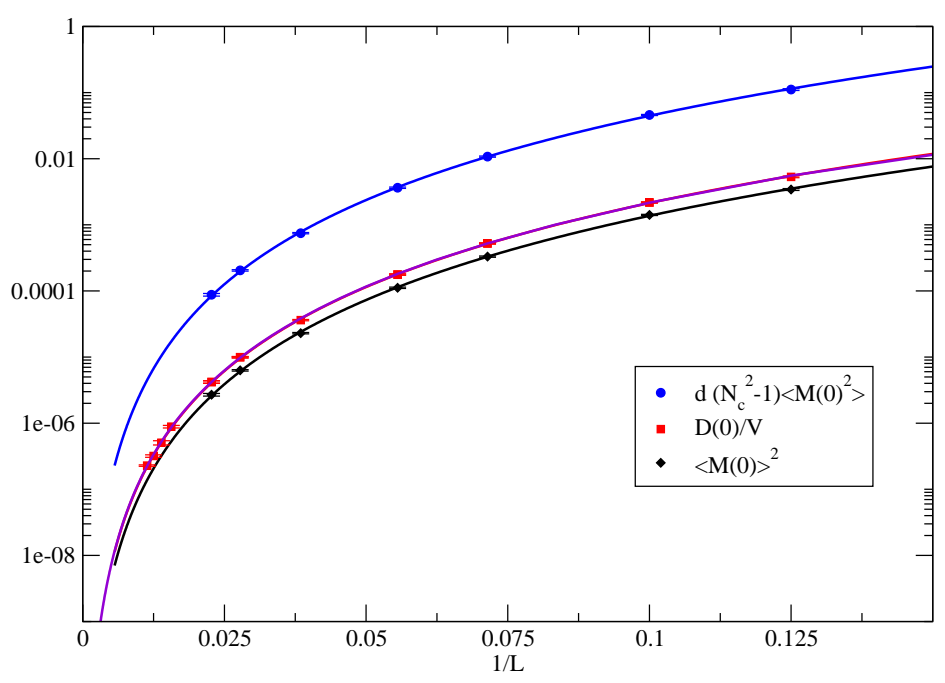

Figure 6: Cucchieri-Mendes bounds, the lattice data and corresponding fits to $A / V^{\alpha}$. Note that in all fits the $26^{4}$ data were excluded in order to have a $\chi^{2} /$ d.o.f. $<1$.8. Furthermore, for $D(0) / V$ the plot includes the fits to the smaller set of lattices, i.e. $8^{4}-44^{4}$ and to all data available, i.e. to the lattices $8^{4}-88^{4}$. The agreement between the two sets of data is perfect.

claimed $D(0) \geq 2.2(3) \mathrm{GeV}^{-2}$. A similar analysis was performed for $\mathrm{SU}(3)$ in [11] and the conclusions favour a $D(0)=0$, although a finite nonvanishing $D(0)$ was not completely excluded. The simulations use the Wilson action and different lattice spacings and volumes. For the SU(2) simulations the authors used $a \sim 0.22 \mathrm{fm}$ and volumes up to $(27 \mathrm{fm})^{4}$, whereas the $\mathrm{SU}(3)$ simulations were performed with $a \sim 0.10 \mathrm{fm}$ and volumes up to $(6.5 \mathrm{fm})^{4}$.

Using the lattice data described before for $\beta=5.7$, we are now in position of review the scaling analysis for the $S U(3)$ gauge theory — see also [12]. Note that for the largest volumes $64^{4}-96^{4}$, which are data from the Berlin-Moscow-Adelaide group, we only have access to $D(0)$.

Anyway, following $[10,11]$, we assume that in (4.1) the different functions depend on the lattice volume as $A / V^{\alpha}$. Then, it follows that an $\alpha>1$ means $D(0)=0$ in the infinite volume. The fits of the lattice data to the small set of volumes $(L \leq 8.1 \mathrm{fm})$ give

\begin{tabular}{cccc}
\hline & $\langle M(0)\rangle^{2}$ & $D(0) / V$ & $\left\langle M(0)^{2}\right\rangle$ \\
\hline$\alpha$ & $1.0537(50)$ & $1.0504(45)$ & $1.0530(50)$ \\
\hline
\end{tabular}

and confirm the conclusions presented in [11], as we found $\alpha>1$. If one wants to use the full set of $\beta=5.7$ lattices, we can only investigate the scaling behaviour of $D(0) / V$. In this case, the fit gives $\alpha=1.0538(28)$ with a $\chi^{2} /$ d.o.f. $=0.87$, which is in excelent agreement with the estimation using the smaller set of lattices, and again it suggests a $D(0)=0$ in the infinite volume. The $\beta=5.7$ lattice data and the corresponding fits to $A / V^{\alpha}$ can be seen in figure 6. Again, as in [11], if one assumes that the dependence with the volume of the different members of (4.1) are given by $C / V+D / V^{\alpha}$, then the data is well described by this functional form; in this sense, a finite and non-vanishing value for $D(0)$ in the infinite volume is not excluded. 


\section{Power-law Behaviours From Ratios Of Propagators}

In [13] a method was proposed which tests the compatibility of the lattice data with a power law behaviour and, simultaneously, suppresses the finite volume effects. Since the method is not wellknown, for completeness, we will review it. The infrared propagator can be investigated using on-axis momenta which are defined as

$$
q[n]=\frac{2}{a} \sin \left(\frac{\pi n}{L}\right), \quad n=0,1, \ldots, \frac{L}{2}
$$

for a symmetry $L^{4}$ lattice. If the gluon propagator is described by a pure power law, i.e.

$$
D\left(q^{2}\right)=Z\left(q^{2}\right)^{2 \kappa-1},
$$

one can define the following ratio

$$
\begin{aligned}
R[n] & =\ln \left[\frac{q^{2}[n+1] D\left(q^{2}[n+1]\right)}{q^{2}[n] D\left(q^{2}[n]\right)}\right] \\
& =2 \kappa R_{q}[n]=2 \kappa \ln \left[\frac{q^{2}[n+1]}{q^{2}[n]}\right] .
\end{aligned}
$$

The compatibility of the lattice data with a power law can be tested fitting the lattice data to

$$
R[n]=2 \kappa R_{q}[n]+C,
$$

Gluon Propagator

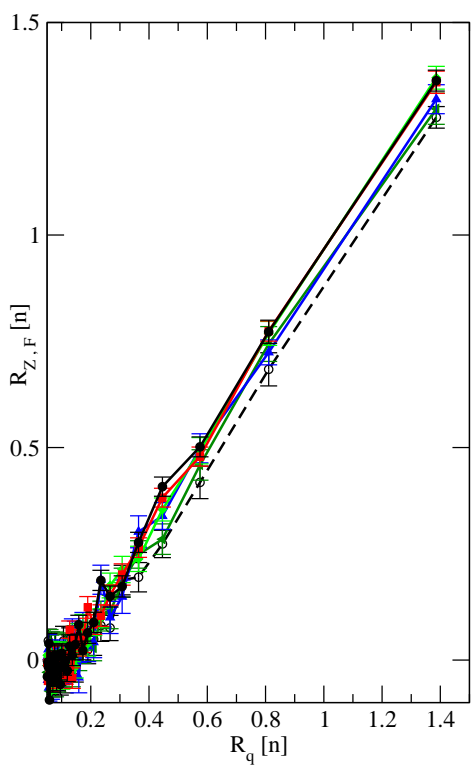

Ghost Propagator

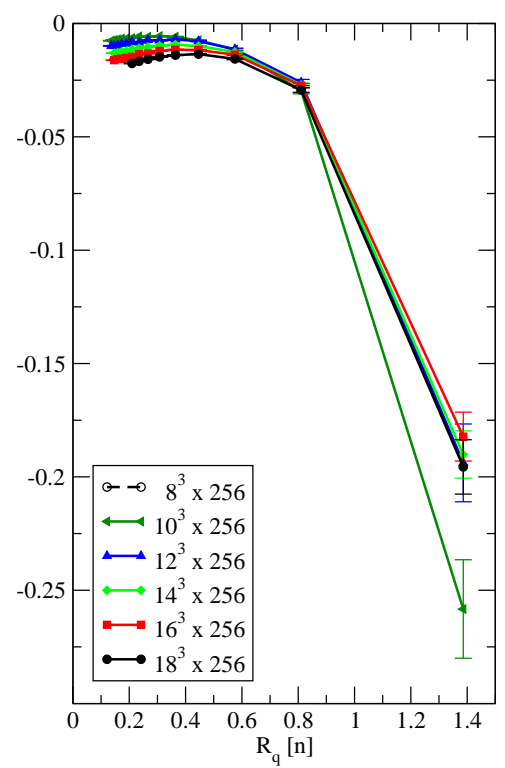

Figure 7: Ratios of gluon and ghost propagators for large asymmetric lattices [13]. Note that, while the gluon data seems to be compatible with a power law behaviour, the ghost propagator does not seem to follow a power law. 


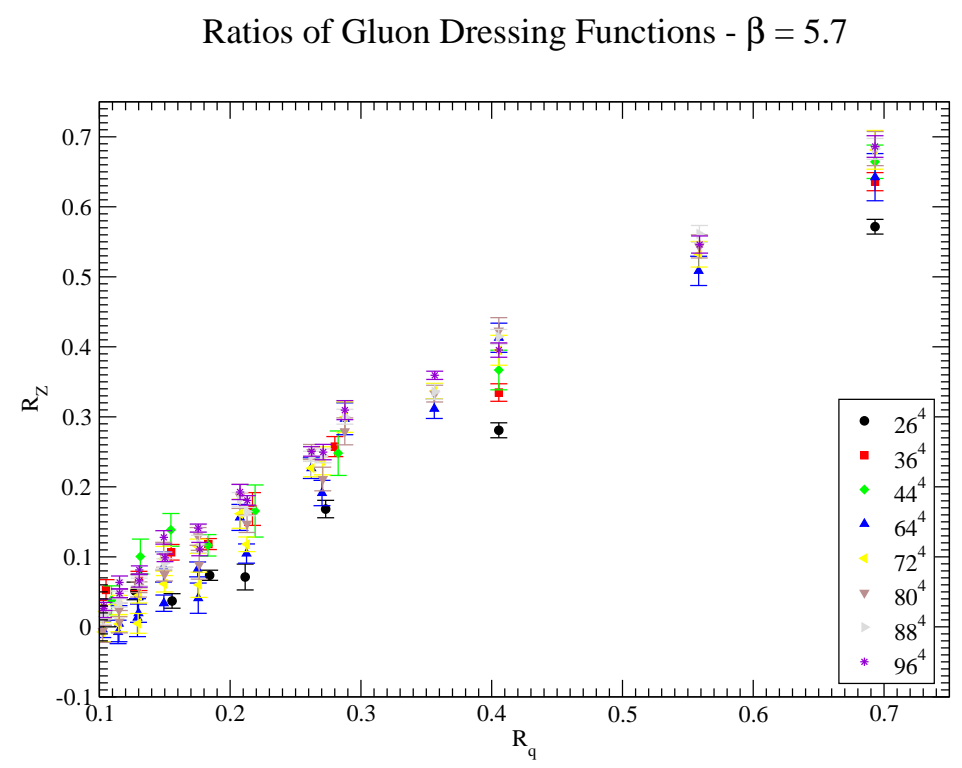

Figure 8: Ratios of propagators for $\beta=5.7$ lattices.

where $C$ is a constant which resumes both the deviations from a power law behaviour and the finite volume effects which are not eliminated by taking the ratio of propagators in $R[n]$. In [13], the authors analyzed such type of fits to the lattice data for large asymmetric $8^{3}-18^{3} \times 256$ lattices - see figure 7. For the gluon propagator the fits give, within one standard deviation, $\kappa>0.5$ with $\kappa \sim 0.53$ and a constant $C$ which seems to approach zero as we go from $8^{3} \times 256$ to $18^{3} \times 256$. The first result suggests a $D(0)=0$, while the second result suggests that $C$ mainly resumes the finite volume effects. In what concerns the ghost propagator, figure 7 shows that either the data is still far from the linear behaviour or the ghost propagator does not follow a pure power law in the infrared region.

The ratios for the $\beta=5.7$ propagators can be seen in figure 8 . We have tried to fit the data coming from the largest lattice volumes to (5.5) but it turns out that the $\chi^{2} /$ d.o.f. were always too large, i.e. well above 2 . This is probably due to rotational invariance violations as the infrared Berlin-Moscow-Adelaide data mixes different types of momenta which have different types of corrections due to the lack of rotational invariance. Indeed a similar effect is seen in results obtained from other lattices when one mixes on-axis with off-axis momenta. Given that we cannot distinguish between the two types of points in the Berlin-Moscow-Adelaide data, we show in figure 9 the ratios for some asymmetric lattices and the large volume $\mathrm{SU}(2)$ propagators. The figure includes the $\kappa$ measured from fitting the lattice data to (5.5). The $128^{4}$ results for the $\mathrm{SU}(2)$ gauge group is the only data which gives a $\kappa<0.5$, within one standard deviation. However, the infrared $128^{4}$ propagator, see figure 10, shows larger fluctuations than any other calculation. Furthermore, it is one of the very few simulations where one sees an enhancing of the propagator in infrared region. Certainly, this is due to the small statistics. 


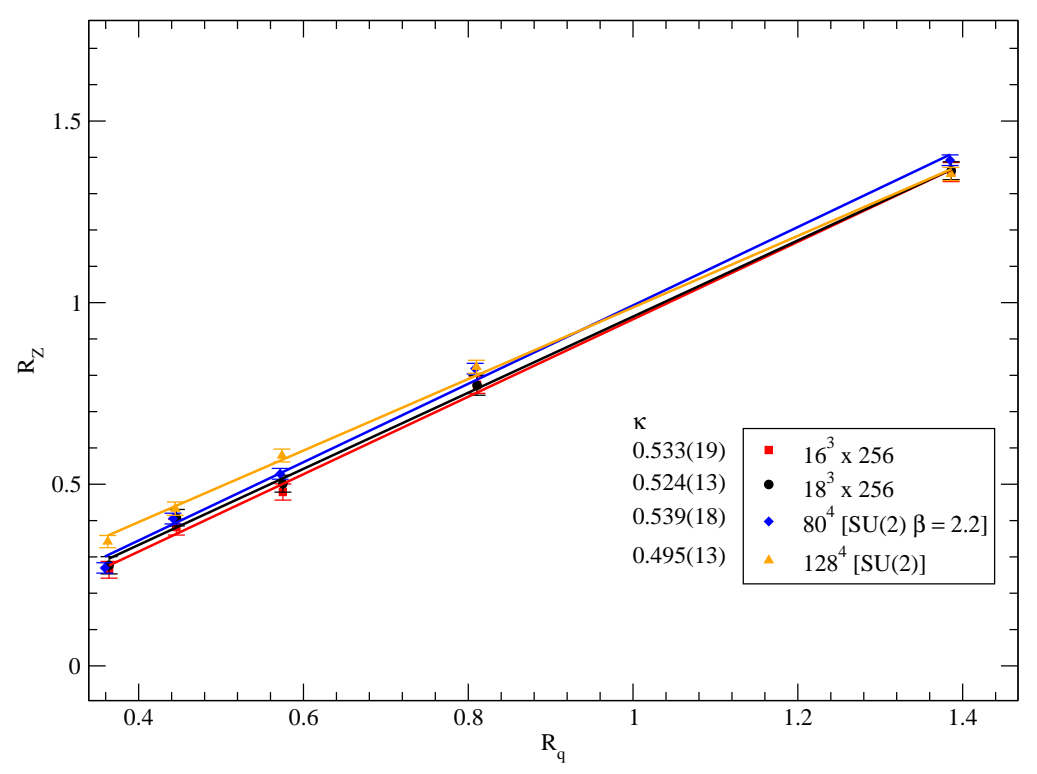

Figure 9: Ratios of propagators for $\mathrm{SU}(3)$ asymmetric lattices at $\beta=6.0$ and $\mathrm{SU}(2)$ simulations.

\section{How Different are the SU(2) and SU(3) Infrared Gluon Propagators?}

As a final topic, we would like to discuss how different are the SU(2) and SU(3) propagators. The discussion of section 3 suggests that there should be a difference in the infrared. Indeed, the mass scales for SU(2) and SU(3) don't seem to be equal with $M_{S U(2)}>M_{S U(3)}$. Moreover, the scaling analysis of the Cucchieri-Mendes bounds seems to give different conclusions when we use different gauge groups - see section 4 and $[10,11]$. However, in $[14,15]$ it was shown that the two propagators are similar for momenta larger than $\sim 800 \mathrm{MeV}$. For smaller momenta, one can see some differences whose origin was not clear. If one uses now the SU(2) data from the S. Carlos group and the $\mathrm{SU}(3)$ Berlin-Moscow-Adelaide data one can compare results for volumes up to $(17 \mathrm{fm})^{4}$. In order to compare the $\mathrm{SU}(2)$ and $\mathrm{SU}(3)$ propagators, they were renormalized by the condition

$$
\left.D\left(q^{2}\right)\right|_{q^{2}=\mu^{2}}=\frac{1}{\mu^{2}}
$$

choosing $\mu=3 \mathrm{GeV}$. The renormalization constants were computed after fitting $D\left(q^{2}\right)$ to the 1loop perturbative result for $q>2.5 \mathrm{GeV}$. In these fits for $\mathrm{SU}(3)$ we used the conic cut data, while for the $\mathrm{SU}(2)$ we used only the diagonal momenta. The renormalization constants were computed from the condition (6.1) using the results of the fits. The statistical error in the renormalization constants being around $10 \%$.

The renormalized propagators, which can be seen in figure 10, show clear differences in the infrared region. This result is not in contradiction with [14], which had a limited access to the low momenta region. Furthermore, reading $D(0)$ from figure 10 it follows that

$$
\frac{D(0)_{S U(2)}}{D(0)_{S U(3)}} \sim \frac{5}{7.5}=\frac{2}{3}
$$




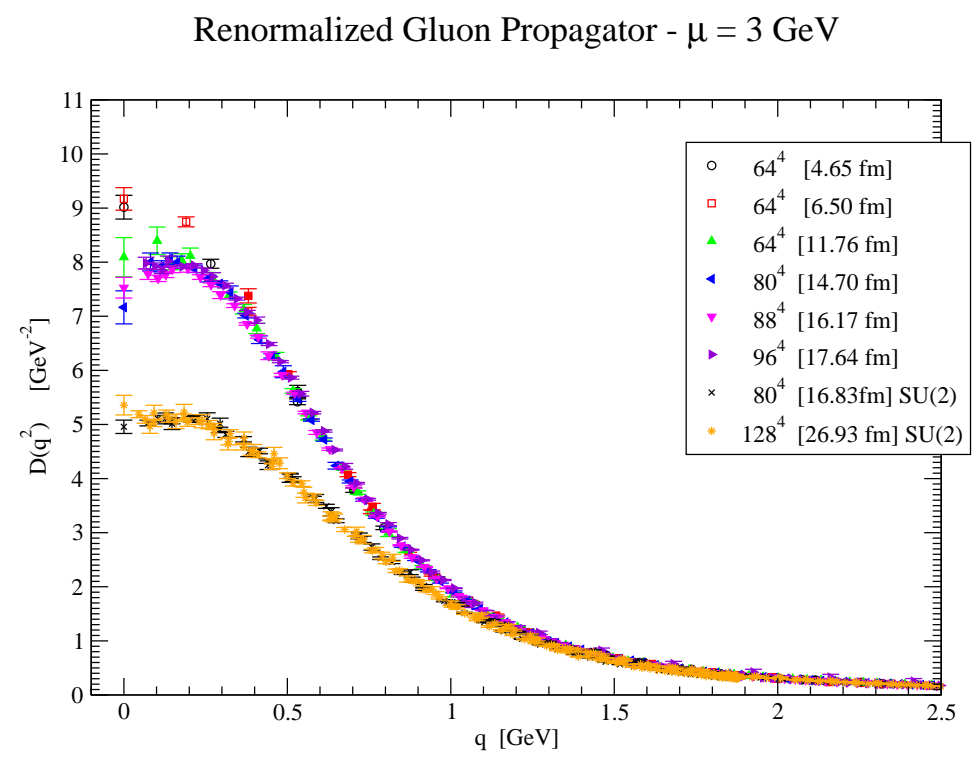

Figure 10: Comparing SU(2) and SU(3) gluon propagators.

suggesting a $D(0)_{S U\left(N_{c}\right)} \sim N_{c}$. This result is not necessarily in conflict with the large $N_{c}$ expansion because the gluon propagator is not a gauge invariant object.

We would like to call the reader's attention that, although the propagators are different, the infrared exponents computed by the ratio method discussed in the previous section seem to be similar.

\section{Conclusions}

The results discussed in this article show that results obtained from lattice simulations for the gluon propagator in Landau gauge can be made compatible with both types of Schwinger-Dyson solutions, i.e. it can be seen either as a scaling like solution, where $D(0)=0$, or as a decoupling solution, where $D(0)$ is finite and non-vanishing. The raw lattice data shows a plateau at small momenta and, in this sense, it does look more like as a decoupling type of propagator.

The analysis of the bounds derived in [10] are not conclusive when applied to the SU(3) simulations $[11,12]$. According to the analysis shown here for the $\beta=5.7$, the leading behaviour is already captured when one includes volumes as "small" as $\sim(8 \mathrm{fm})^{4}$. Moreover, the performed scaling analysis is in good agreement with the ratios analysis, favouring a $D(0)=0$. This does not necessarily mean that the lattice simulations point towards a scaling like solution. Remember that the ratio method applied to the asymmetric lattice data shows that the ghost propagator does not follow a power law. In what concerns the gluon and ghost propagators, it looks like that before having a clear understanding of the finite lattice volume/spacing effects, it will be quite difficult to give a definitive answer on the nature of the propagators in the deep infrared region.

Finally, the Landau gauge gluon $\mathrm{SU}(2)$ and $\mathrm{SU}(3)$ propagators are compared for momenta below $800 \mathrm{MeV}$. It turns out that there are clear differences and a scaling law $D(0)_{S U\left(N_{c}\right)} \sim N_{c}$ seems to hold. 


\section{Acknownledgements}

We would like to thank the Berlin-Moscow-Adelaide and S. Carlos groups for sending us their data and for allowing to use it.

The authors also acknowledge financial support from FCT under projects CERN/FP/83582/2008 and CERN/FP/83664/2008. P. J. S. was supported by FCT via grant SFRH/BPD/40998/2007.

\section{References}

[1] C. Lerche, L. von Smekal, Phys. Rev. D65, 125006 (2002).

[2] C.S.Fischer, J. Phys. G32, R253 (2006), arXiv: hep-ph/ 0605173.

[3] A. C. Aguilar, D. Binosi, J. Papavassiliou, Phys. Rev. D78, 025010 (2008) arXiv : 0802 . 1870 [hep-ph] .

[4] D. Binosi, J. Papavassiliou, Phys. Rep. 479, 1 (2009), arXiv:0909.2536 [hep-ph].

[5] D. Dudal, J.A. Gracey, S.P. Sorella, N. Vandersickel, H. Verschelde, Phys. Rev. D78, 065047 (2008).

[6] P. J. Silva, O. Oliveira, Nucl. Phys. B690, 177 (2004), arXiv: hep-lat / 0403026.

[7] G. S. Bali, K. Schiling, Phys. Rev. D47, 661 (1993), arXiv: hep-lat/ 9208028.

[8] I. L. Bogolubsky, E.-M. Ilgenfritz, M. Müller-Preusker, A. Sternbeck, Phys. Lett. B676, 69 (2009), arXiv:0901.0736 [hep-lat].

[9] A. Cucchieri, T. Mendes, PoS (LAT2007) 297, arXiv:0710.0344 [hep-lat].

[10] A. Cucchieri, T. Mendes, Phys. Rev. Lett. 100, 241601(2008), arXiv:0712.3517 [hep-lat ].

[11] O. Oliveira, P. J. Silva, Phys. Rev. D79, 031501(R) (2009), arXiv: 0809.0258 [hep-lat ].

[12] O. Oliveira, P. J. Silva, PoS (LAT2009) 226, arXiv:0910.2897 [hep-lat].

[13] O. Oliveira, P. J. Silva, EPJ C62, 525 (2009), arXiv: 0705.0964 [hep-lat]; PoS (LATTICE 2007) 332, arXiv:0710.0665 [hep-lat].

[14] A. Cucchieri, T. Mendes, O. Oliveira, P. J. Silva, Phys. Rev. D76, 114507 (2007), arXiv:0705.3367 [hep-lat].

[15] A. Sternbeck, L. von Smekal, D. B. Leinweber, A. G. Williams, PoS (LAT2007), 340 (2007), arXiv:0710.1982 [hep-lat]. 